\title{
Quantum Oscillations on the Surface of InAs Epilayer
}

\author{
Yahua Yuan ${ }^{\mathrm{a}}$, Xuhui Wang ${ }^{\mathrm{b}}$, Jurgen Kosel $^{\mathrm{c}}$, Jian Sun ${ }^{\mathrm{a}, *}$ \\ ${ }^{a}$ School of Physics and Electronics, Central South University, 932 South Lushan Road, \\ Changsha 410083, China \\ ${ }^{b}$ Kwantum Links, Benoordenhoutseweg 23, 2596 BA Den Haag, The Netherlands \\ ${ }^{c}$ Computer, Electrical and Mathematical Sciences and Engineering Division, King Abdullah \\ University of Science and Technology (KAUST), Thuwal 23955-6900, Saudi Arabia
}

\begin{abstract}
Indium arsenide (InAs), a narrow-gap semiconductor, has a highly conductive 2-dimensional surface state naturally formed as a result of band bending at the free surface. The Shubnikov-de Haas oscillations have been studied widely in its heterostructures, e.g. 2-dimensional electron gases. However, studies on such a nature surface state are missing. Here, we report a Shubnikov-de Haas ( $\mathrm{SdH})$ oscillation that originates from the InAs surface state. Two leading oscillation frequencies in the $\mathrm{SdH}$ signal are attributed to Rashba spin-orbit coupling residing in the surface state. We also found for the surface state an effective electron mass of $0.038 m_{0}$, heavier than $\sim 0.023 m_{0}$ in the bulk. Our study also suggests a Rashba coupling constant in the order of $\sim 10^{-11} \mathrm{eV} \cdot \mathrm{m}$, showing good agreement with previously reported values for InAs.

Keywords: Indium arsenide, Surface state, Shubnikov-de Haas oscillation, Berry phase, Rashba spin-orbit coupling
\end{abstract}

\section{Introduction}

With a robust spin-orbit interaction [1, 2], indium arsenide (InAs) is a narrow-gap semiconductor that has promising spintronic applications. On its free surface, a two-dimensional (2D) charge accumulation with a sheet density

\footnotetext{
* Corresponding author

Email address: jian.sun@csu.edu.cn (Jian Sun)
}

Preprint submitted to Physica E: Low-dimensional Systems and Nanostructures June 12, 2019 
5 of $\sim 10^{12} \mathrm{~cm}^{-2}$ is naturally formed, due to band bending. This essential property has been revealed previously by angle-resolved photoemission spectroscopy [3]. As the potential gradient across the vacuum-InAs interface produces an electric field that is normal to the surface, the electron with a wave-vector $\boldsymbol{k}$ is subject to the Bychkov-Rashba spin-orbit coupling $\alpha \hat{\boldsymbol{\sigma}} \cdot(\boldsymbol{k} \times \hat{\boldsymbol{z}})[4] . \alpha$ is the

10 Rashba coupling constant, $\hat{\boldsymbol{z}}$ is the unit vector of the surface normal, and $\hat{\boldsymbol{\sigma}}$ is the Pauli matrix. This coupling between charge and spin has been scrutinised in various systems, ranging from conventional semiconductors [5, 6] to topological insulators [7].

Shubnikov-de Haas ( $\mathrm{SdH}$ ) oscillation in magnetoconductance is a well-known 15 phenomenon that reveals the energy dispersion and Fermi surface properties of the underlying system [8]. The oscillatory part of the longitudinal magnetoconductance $\Delta G_{x x}$ depends on the magnetic field $B$

$$
\Delta G_{x x} \sim \cos \left[2 \pi\left(\frac{f_{\mathrm{SdH}}}{B}+\frac{\Gamma}{2 \pi}-\frac{1}{2}\right)\right],
$$

where $f_{\mathrm{SdH}}$ is the oscillation frequency, and $\Gamma$ is the so-called Berry phase [9]. As a geometric phase, Berry phase in a solid state system is determined by the topology of the energy bands [10]. A nonzero Berry phase $(\Gamma=\pi)$ can be achieved by creating a band touching or Dirac point [11]. This has been observed in many 2D systems such as graphene [9, 12], the surface states in topological insulators [13, 14], topological Weyl semimetal [15], and three-dimensional Dirac semimetal [16]. The SdH oscillations have been widely investigated in varied 25 two-dimensional electron gases of InAs in the past decades [17, 18, 19, 20, 21. However, We are not aware of any sophisticated measurements of the SdH oscillations in the surface state on top of a bulk InAs.

In this paper, we report a magnetotransport measurement and the observation of $\mathrm{SdH}$ oscillation in an $\mathrm{n}$-doped InAs epilayer. The data verifies the so existence of a 2D electron gas on the InAs surface. A Fourier analysis on the oscillation signal shows two frequencies that are a result of two different Fermi circles created by the Rashba-type coupling. Furthermore, Berry phase is roughly estimated from the Landau level fan diagram and it has a nonzero 

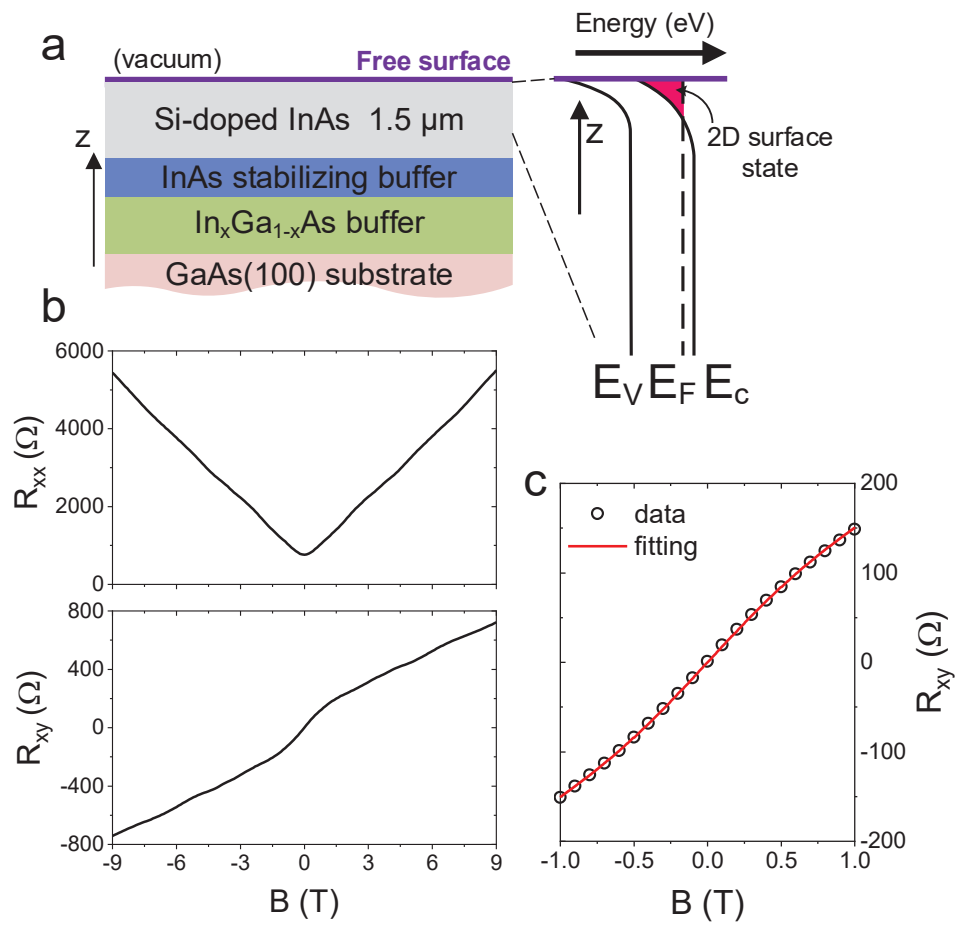

Figure 1: (Color online)(a) Layer diagram showing the n-doped InAs epitaxial structure and a schematic of the formation of $2 \mathrm{D}$ surface state due to band bending. (b) Magnetotransport in the InAs epilayer. Longitudinal Magnetoresistance $R_{x x}$ and Hall resistance $R_{x y}$ in versus magnetic field at $10 \mathrm{~K}$. (c) Fitting of the two-layer model to the low-field Hall data in (b).

value. All these results are consistent with the intrinsic features of a $2 \mathrm{D}$ electron gas that is submitted to a Rashba-type spin-orbit coupling. The Rashba constant of $\alpha \sim 10^{-11} \mathrm{eV} \cdot \mathrm{m}$ is extracted which is consistent with the typical values reported for InAs previously.

\section{Experiments}

The InAs sample used in this work is a 1.5- $\mu$ m-thick, Si-doped epilayer grown

40 on a (100) oriented semi-insulating GaAs substrate using solid-source molecular beam epitaxy, see Fig.1 (a). The compensation effect is negligible with a doping level of $10^{16} \mathrm{~cm}^{-3}$. The growth started with a 1- $\mu$ m-thick $\operatorname{In}_{x} \mathrm{Ga}_{1-x}$ As meta- 
morphic buffer to accommodate the large lattice mismatch between InAs and GaAs, which was followed by a $0.2-\mu$ m-thick undoped InAs stabilizing buffer. Photo-lithographic techniques were used for device patterning. The InAs bar of size $40 \mu \mathrm{m} \times 1000 \mu \mathrm{m}$ was wet etched in a citric acid $/ \mathrm{H}_{2} \mathrm{O}_{2}$ solution exploiting the semi-insulating GaAs as an etch stop. The contacts were formed by sputtered $\mathrm{Ti}(50 \mathrm{~nm}) / \mathrm{Au}(250 \mathrm{~nm})$. A rapid thermal annealing process at $250{ }^{\circ} \mathrm{C}$ was utilized to improve the contact. The ohmic contact was then confirmed by the linear current-voltage response. The magnetotransport measurements were carried out with a four-probe geometry in a Quantum Design physical property measurement system (PPMS) with a magnetic field up to 9 T. A constant current of $100 \mu \mathrm{A}$ was applied to the device throughout the measurements.

\section{Results and discussion}

We show in Fig.1(b) the longitudinal magnetoresistance $R_{x x}$ and Hall resistance $R_{x y}$ acquired at $10 \mathrm{~K}$. The nonlinear Hall resistance $R_{x y}$ is due to parallel conducting channels [22, 23], namely, the coexistence of the surface and the underlying bulk. In order to single out the contribution of the surface state to the overall transport, we fit the data of $R_{x y}$ by a two-layer model, see Fig.11(c),

$$
R_{x y}(B)=\frac{B\left(\mu_{s} G_{s}+\mu_{b} G_{b}\right)+B^{3}\left(\mu_{b}^{2} \mu_{s} G_{s}+\mu_{s}^{2} \mu_{b} G_{b}\right)}{\left(G_{s}+G_{b}\right)^{2}+B^{2}\left(\mu_{s} G_{b}+\mu_{b} G_{s}\right)^{2}} .
$$

60

In the above, the conductance $G_{b(s)}=e n_{b(s)} \mu_{b(s)} d_{b(s)}$ is determined by the carrier density $n$, mobility $\mu$, and thickness $d$ [22, 23]. The subscript $b(s)$ refers to the bulk (surface) layer. The best fitting suggests a carrier density $n_{s} \sim 1.50 \times 10^{18} \mathrm{~cm}^{-3}$. This translates into an equivalent sheet density $N_{s} \sim$ $1.48 \times 10^{12} \mathrm{~cm}^{-2}$ in the surface, and $n_{b} \sim 2.35 \times 10^{16} \mathrm{~cm}^{-3}$ in the bulk. The order of magnitude of the sheet density estimated here agrees with the previously reported value [3], and the bulk density is consistent with the Si-doping level of $10^{16} \mathrm{~cm}^{-3}$.

As the magnetic field scan extends to a higher field, a weak oscillation, although barely visible, starts to emerge in the resistance. In order to gain correct 

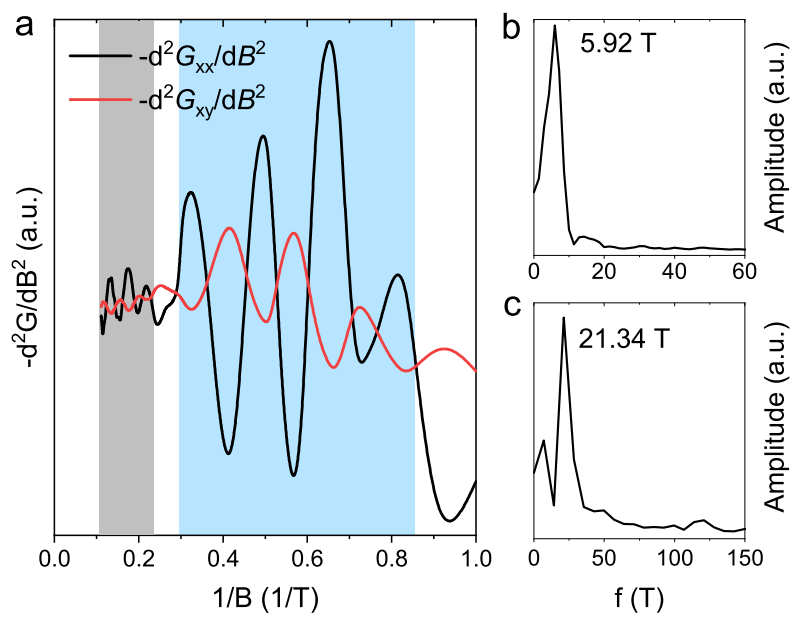

Figure 2: (a) Quantum oscillations in $-d^{2} G_{x x} / d B^{2}$ and $-d^{2} G_{x y} / d B^{2}$ in InAs. (b) Fourier analysis on the $-d^{2} G_{x x} / d B^{2}$ signal at low field (blue zone) and (c) at high field (grey zone).

information on the carrier density and Berry phase from the oscillatory data, we first convert the resistance to conductance following a standard procedure [24]. Then, we can extract the oscillatory signal by calculating $-d^{2} G_{x x} / d B^{2}$ and $-d^{2} G_{x y} / d B^{2}$ and they are shown in Fig.2(a). As functions of the inverse field $1 / B$, both oscillations show strong periodicity. The phase shift between ${ }_{75}-d^{2} G_{x x} / d B^{2}$ and $-d^{2} G_{x y} / d B^{2}$ is approximately $\sim \pi$, instead of $\pi / 2$. A similar phase difference of $\pi$ has been reported before in a topological insulator [23]. This is attributed to the coexistence of both surface and bulk conducting channels. Note here the second derivative only carries the information of frequency, while its amplitude does not correspond to real signal of the conductance change $-\Delta G_{x x}$.

Using Fourier analysis on $-d^{2} G_{x x} / d B^{2}$, we find two distinct frequencies that are dominating in the $\mathrm{SdH}$ signal in low and high magnetic fields, respectively. In the field below $\sim 3 \mathrm{~T}$ (called low-field regime thereafter), a frequency $f_{\mathrm{SdH}}^{(\mathrm{L})}=$ $5.92 \mathrm{~T}$ is identified in Fig.2(b). In the field higher than $\sim 4 \mathrm{~T}$ (called high field regime thereafter), see Fig. 3(c), a frequency of $21.34 \mathrm{~T}$ is read out from the analysis. The ratio $f_{\mathrm{SdH}}^{(\mathrm{H})} / f_{\mathrm{SdH}}^{(\mathrm{L})}$ is around 3 . 
We first uncover the origin of these oscillations. If the oscillations were originating from the bulk, the carrier contribution would be $4.72 \times 10^{18} \mathrm{~cm}^{-3}$, using $e f_{\mathrm{SdH}} /(c \hbar \pi)$, with $c=6.06 \AA$ being the lattice constant. However, the designed bulk Si-doping level $\left(10^{16} \mathrm{~cm}^{-3}\right)$ and the bulk carrier density we acquire from the Hall measurement $\left(2.35 \times 10^{16} \mathrm{~cm}^{-3}\right)$ is - by two orders of magnitude - lower than this. This compels us to argue that it is unlikely that these SdH oscillations are originating from the bulk, and hence the SdH signal mainly arises from the $2 \mathrm{D}$ surface state.

The presence of two dominating frequencies in the $\mathrm{SdH}$ signal provides an evidence to the existence of two well-defined Fermi circles in the 2D surface state. So far, all experimental features are consistent with the 2D BychkovRashba model. In order to estimate the Rashba constant $\alpha$ for the surface, we convert the higher frequency $f_{\mathrm{SdH}}^{(\mathrm{H})}=\hbar\left(k_{F}^{(-)}\right)^{2} /(2 e)$ and the lower one $f_{\mathrm{SdH}}^{(\mathrm{L})}=$ $\hbar\left(k_{F}^{(+)}\right)^{2} /(2 e)$ to the Fermi wave vectors $k_{F}^{( \pm)}$that are determined by the two polarization branches due to the Rashba splitting, i.e. $E_{F}=\hbar^{2}\left(k_{F}^{( \pm)}\right)^{2} /\left(2 m^{*}\right) \pm$ $\alpha k_{F}^{( \pm)}$. By estimating the Fermi energy from the total carrier density $N_{s}$, the leading order of $\alpha$ follows from

$$
f_{\mathrm{SdH}}^{(\mathrm{H})}-f_{\mathrm{SdH}}^{(\mathrm{L})} \approx 2 \frac{\alpha m^{*}}{e \hbar} \sqrt{2 \pi N_{s}},
$$

where the Fermi energy is assumed to be larger than the Rashba energy $\Delta_{\mathrm{R}} \equiv$ $\alpha^{2} m^{*} /\left(2 \hbar^{2}\right)$.

We estimate the carrier density by using the spin-filtered Onsager relation $N_{s}=(e / \pi \hbar) f_{\mathrm{SdH}}$. In the low field regime, $f_{\mathrm{SdH}}^{(\mathrm{L})}$ leads to a sheet density $N_{s}=2.86 \times 10^{11} \mathrm{~cm}^{-2}$. And in the high field regime, the frequency of $21.34 \mathrm{~T}$ corresponds to $N_{s} \sim 1.03 \times 10^{12} \mathrm{~cm}^{-2}$.

The effective mass $m^{*}$ of the surface state can be extracted from the temperature dependence of the $\mathrm{SdH}$ oscillation. The magnetoresistance $R_{x x}$ is measured at various temperatures from 8 to $16 \mathrm{~K}$. The oscillatory component $\Delta R_{x x}$, originating from the surface, is plotted in Fig. 3(a). The background - a rather smooth signal - is removed by fitting the data into a fourth-order polynomial. 115 This method is similar to the one used by Murakawa et al. [25]. The oscillation 

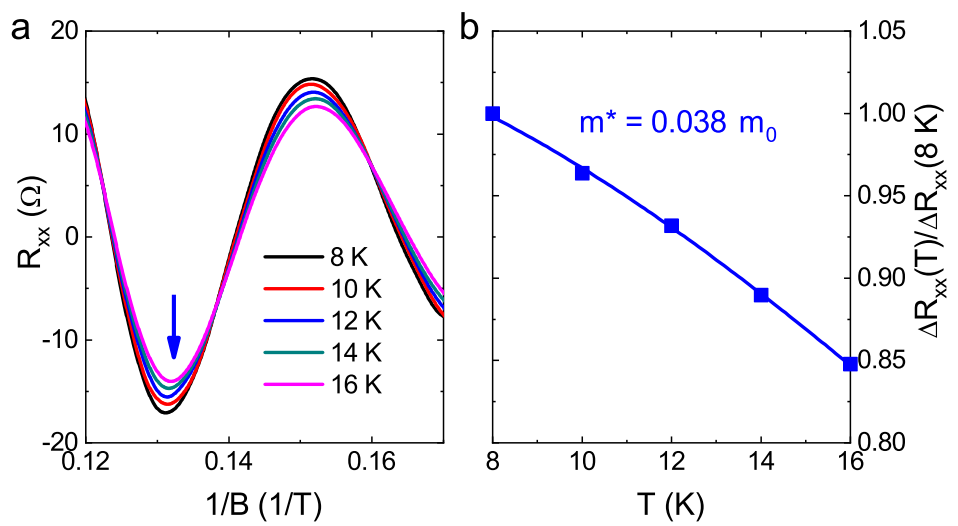

Figure 3: (a) Oscillatory component $\Delta R_{x x}$ obtained for the oscillations measured in InAs at various temperatures from 8 to $16 \mathrm{~K}$. Blue arrows point out the location of $7.4 \mathrm{~T}$. (b) Temperature dependence of the normalized SdH oscillation amplitudes at $7.4 \mathrm{~T}$. The solid line is the best fits to Eq.(4)

amplitude decreases with increasing temperature $T$. The $T$ dependence of the SdH oscillation amplitude of $R_{x x}$ obeys the Lifshits-Kosevich formula,

$$
\frac{\Delta R_{x x}(T)}{\Delta R_{x x}\left(T_{0}\right)}=\exp \left(-\frac{\lambda T_{D} m^{*}}{B_{n}}\right) \frac{\lambda T m^{*} / B_{n}}{\sinh \left(\lambda T m^{*} / B_{n}\right)},
$$

where $\Delta R_{x x}(T) / \Delta R_{x x}\left(T_{0}\right)$ is the normalized oscillatory component and $\lambda=$ $2 \pi^{2} k_{B} / \hbar e$. By fitting the temperature dependence of the oscillatory component 120 to Eq.(何), $m^{*}$ is obtained, as shown in Fig. 3(b). At magnetic field of 7.6 T, the fitting yields effective masses $m^{*}$ of $0.038 m_{0}$. This value is in good agreement with $0.033 m_{0}$ reported for the InAs surface in an earlier paper [26]. For a comparison, the effective mass is about $0.023 m_{0}$ in bulk InAs. From Eq. (3), the Rashba constant in the surface state can be calculated as $\alpha \sim 7.3 \times 10^{-11} \mathrm{eV} \cdot \mathrm{m}$.

${ }_{125}$ This value is large and in the same order of magnitude compared to that in the typical InAs 2D systems [5, 21].

In principle, the free surface of InAs shall support a nonzero Berry phase. Its built-in Rashba-type coupling may introduce in the energy dispersion a $k$ linear term in the neighbourhood of $k \sim 0$ [ 4$]$. To extract the Berry phase $\Gamma$, we construct the Landau level fan diagram from the SdH oscillation data [9, 23]. A minimum in the oscillating conductance is translated to a complete filling up 


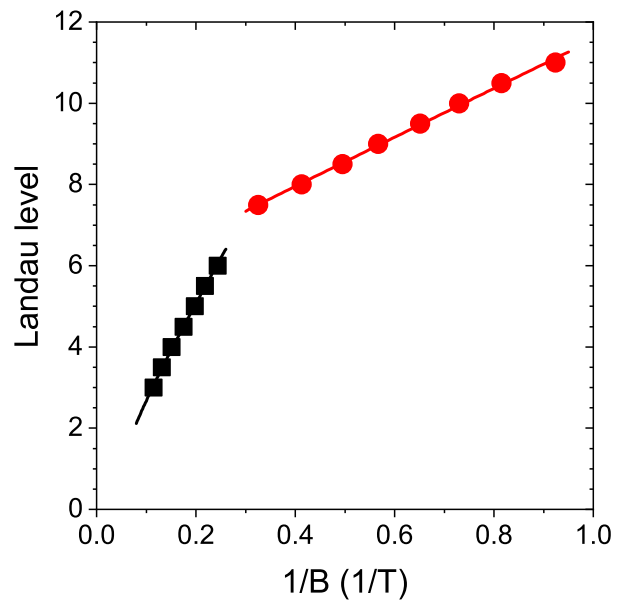

Figure 4: Landau level fan diagram constructed from $-d^{2} G_{x x} / d B^{2}$ data exhibited in Fig. 2a. The solid lines are the fittings in separate regimes using Eq. 5. Values of $f_{\mathrm{SdH}}$ are fixed at the respective frequencies obtained from Fourier analysis.

to the $N$ th Landau level. In this case, the Fermi energy $E_{F}$ is located between the $N$ th and $(N+1)$ th levels. The values of $B_{N}$ at the minima (maxima) of $-d^{2} G_{x x} / d B^{2}$ are indexed by an integer (a half integer), as shown in Fig. 4 . A

fold point appears at $\sim 3 \mathrm{~T}$. This implies the existence of an internal magnetic field in the surface state [27, 28, 29, 30] and provides another evidence to the presence of a Rashba spin-orbit coupling. A similar picture was reported by Tsukazaki et al. in a two-dimensional electron gas [28]. However, we note that in the high field regime the Landau level plot is not linear, impeding an accurate extraction of the Berry phase. For such a two-band system described by the Bychkov-Rashba model, the calculation of $\Gamma$ requires the energy correction from the coupling between the magnetic field and orbital magnetic moment of the Bloch electrons [10]. With the corrected method to extrapolate $\Gamma$ from $\mathrm{SdH}$ signal, we could fit our Landau plot to [31]

$$
N=\frac{f_{\mathrm{SdH}}}{B_{N}}-\frac{\Gamma}{2 \pi}+\xi B_{N} .
$$

The fitting provides us a rough estimation on the value of Berry phase. At low field, the frequency $f_{\mathrm{SdH}}=5.92 \mathrm{~T}$ yields $\Gamma / 2 \pi=5.56$. This half-integer 
value means that $\Gamma \sim \pi$ at low field and suggests that the system may possess a nonzero Berry phase. At high field, the Zeeman splitting becomes dominant and leads to $\Gamma / 2 \pi=0.06$ and therefore $\Gamma \sim 0$, i.e., a trivial Berry phase. This is consistent with the physical picture outlined by a Bychkov-Rashba model.

\section{Conclusions}

In summary, we have investigated the $\mathrm{SdH}$ oscillation in the naturally formed surface state on the bulk of InAs. Two dominating frequencies extracted from the oscillations indicate the existence of two Fermi circles as a result of the

Rashba spin-orbit coupling in the $2 \mathrm{D}$ surface state. We extract from the data a Rashba coupling constant $\alpha \sim 10^{-11} \mathrm{eV} \cdot \mathrm{m}$. Our results attempt us to explore potential spintronic applications of the InAs surface state and the possibility of tuning an ordinary semiconductor into a pseudo topological insulator.

\section{Acknowledgement}

This work was supported by National Natural Science Foundation of China (Grant No. 11804397).

\section{References}

[1] A.E. Hansen, M.T. Björk, C. Fasth, C. Thelander, L. Samuelson, Phys. Rev. B. 71 (2005) 205328. doi:10.1103/PhysRevB.71.205328.

[2] C. Fasth, A. Fuhrer, L. Samuelson, V.N. Golovach, D. Loss, Phys. Rev. Lett. 98 (2007) 266801. doi:10.1103/PhysRevLett.98.266801.

[3] L.Ö. Olsson, C.B.M. Andersson, M.C. Håkansson, J. Kanski, L. Ilver, U.O. Karlsson, Phys. Rev. Lett. 76 (1996) 3626-3629. doi:10.1103/PhysRevLett.76.3626. 
[4] Y.A. Bychkov, E.I. Rashba, J. Phys. C: Solid State Phys. 17 (1984) 60396045. doi:10.1088/0022-3719/17/33/015.

[5] J. Nitta, T. Akazaki, H. Takayanagi, T. Enoki, Phys. Rev. Lett. 78 (1997) 1335-1338. doi:10.1103/PhysRevLett.78.1335.

175 [6] J.W.G. van den Berg, S. Nadj-Perge, V.S. Pribiag, S.R. Plissard, E.P.A.M. Bakkers, S.M. Frolov, L.P. Kouwenhoven, Phys. Rev. Lett. 110 (2013) 066806. doi:10.1103/PhysRevLett.110.066806.

[7] M.Z. Hasan, C.L. Kane, Rev. Mod. Phys. 82 (2010) 3045-3067. doi:10.1103/RevModPhys.82.3045.

[8] Shoenberg D 1984 Magnetic Oscillations in Metals (Cambridge, UK: Cambridge University Press)

[9] Y. Zhang, Y.-W. Tan, H.L. Stormer, P. Kim, Nature. 438 (2005) 201-204. doi:10.1038/nature04235.

[10] D. Xiao, M.-C. Chang, Q. Niu, Rev. Mod. Phys. 82 (2010) 1959-2007. doi:10.1103/RevModPhys.82.1959.

[11] G.P. Mikitik, Yu.V. Sharlai, Phys. Rev. Lett. 82 (1999) 2147-2150. doi:10.1103/PhysRevLett.82.2147.

[12] K.S. Novoselov, E. McCann, S.V. Morozov, V.I. Fal'ko, M.I. Katsnelson, U. Zeitler, D. Jiang, F. Schedin, A.K. Geim, Nature Physics. 2 (2006) 177-180. doi:10.1038/nphys245.

[13] D.-X. Qu, Y.S. Hor, J. Xiong, R.J. Cava, N.P. Ong, Science. 329 (2010) 821-824. doi:10.1126/science.1189792.

[14] J.G. Analytis, R.D. McDonald, S.C. Riggs, J.-H. Chu, G.S. Boebinger, I.R. Fisher, Nature Physics. 6 (2010) 960-964. doi:10.1038/nphys1861.

${ }_{195}$ [15] X. Huang, L. Zhao, Y. Long, P. Wang, D. Chen, Z. Yang, H. Liang, M. Xue, H. Weng, Z. Fang, X. Dai, G. Chen, Phys. Rev. X. 5 (2015) 031023. doi:10.1103/PhysRevX.5.031023. 
[16] L.P. He, X.C. Hong, J.K. Dong, J. Pan, Z. Zhang, J. Zhang, S.Y. Li, , Phys. Rev. Lett. 113 (2014) 246402. doi:10.1103/PhysRevLett.113.246402.

200

[17] D. Grundler, Phys. Rev. Lett. $84 \quad$ (2000) 6074-6077. doi:10.1103/PhysRevLett.84.6074.

[18] T. Matsuyama, R. Kürsten, C. Meißner, U. Merkt, Phys. Rev. B. 61 (2000) 15588-15591. doi:10.1103/PhysRevB.61.15588.

[19] J. Nitta, T. Akazaki, H. Takayanagi, T. Enoki, Physica E:

[20] J. Luo, H. Munekata, F.F. Fang, P.J. Stiles, Phys. Rev. B. 41 (1990) 76857693. doi:10.1103/PhysRevB.41.7685.

[21] A.J.A. Beukman, F.K. de Vries, J. van Veen, R. Skolasinski, M. Wimmer,

[24] J. Xiong, Y. Luo, Y. Khoo, S. Jia, R.J. Cava, N.P. Ong, Phys. Rev. B. 86 (2012) 045314. doi:10.1103/PhysRevB.86.045314.

[25] H. Murakawa, M.S. Bahramy, M. Tokunaga, Y. Kohama, C. Bell, Y. Kaneko, N. Nagaosa, H.Y. Hwang, Y. Tokura, Science. 342 (2013) 1490220

[26] L. Canali, J.W.F Wildöer, O. Kerkhof, L.P Kouwenhoven, Appl. Phys. A. 66 (1998) S113. 
[27] J.P. Eisenstein, H.L. Störmer, V. Narayanamurti, A.C. Gossard, W. Wiegmann, Phys. Rev. Lett. 53 (1984) 2579-2582. doi:10.1103/PhysRevLett.53.2579.

[28] A. Tsukazaki, A. Ohtomo, T. Kita, Y. Ohno, H. Ohno, M. Kawasaki, Science. 315 (2007) 1388-1391. doi:10.1126/science.1137430.

[29] H.L. Stormer, Z. Schlesinger, A. Chang, D.C. Tsui, A.C. Gossard, W. Wiegmann, Phys. Rev. Lett. 51 (1983) 126-129. ${ }_{230}$ doi:10.1103/PhysRevLett.51.126.

[30] S.J. Papadakis, E.P.D. Poortere, H.C. Manoharan, M. Shayegan, R. Winkler, Science. 283 (1999) 2056-2058. doi:10.1126/science.283.5410.2056.

[31] A.R. Wright, R.H. McKenzie, Phys. Rev. B. 87 (2013) 085411. doi:10.1103/PhysRevB.87.085411. 